\title{
A new software for fragment-based QSAR and its applications
}

\author{
Sergey B Sosnin*, Vladimir A Palyulin, Nikolay S Zefirov \\ From 9th German Conference on Chemoinformatics \\ Fulda, Germany. 10-12 November 2013
}

Fragment-based methods are quite popular in 2D QSAR/QSPR studies. In the advanced versions of these approaches for developing highly predictive models, one have to generate a huge set of descriptors that in turn requires well-designed algorithms and high-quality parallelism. To overcome these problems we developed the software for tagged generation of fragmental descriptors.

One of the most perspective programming paradigms is functional programming. Programs in pure functional languages are easy to parallelize, tend to be faultless, more clear, easy extensible [1]. Despite its tangible complexity for learning, functional programming is becoming rather popular, recently several chemoinformatics frameworks: OUCH and chemf were presented. We developed the program QLab for the fragmentation of molecular graphs in the imperative programming language (Java, using CDK), and ported it under the name FragmenT into the functional language (Haskell). Aware of the fact that graph operations is a core of chemoinformatics, FragmenT represents chemical structures by means of Functional Graph Library - a powerful tool for graph operations [2].

Using this software we processed several structureactivity/property databases containing hundreds of compounds and generated the sets of more than 500000 fragmental descriptors in each case. Then for each database 100 most correlated with activity/property descriptors were selected using stagewise MLR. Based on selected descriptors the predictive models were developed using artificial neural networks (ANN) and Random Forest algorithms. Comparison with other fragmental descriptors generation software showed better predictive ability of our models.

\footnotetext{
* Correspondence: serg.sosnin@gmail.com

Department of Chemistry, Lomonosov Moscow State University, Moscow,
} 119991, Russia

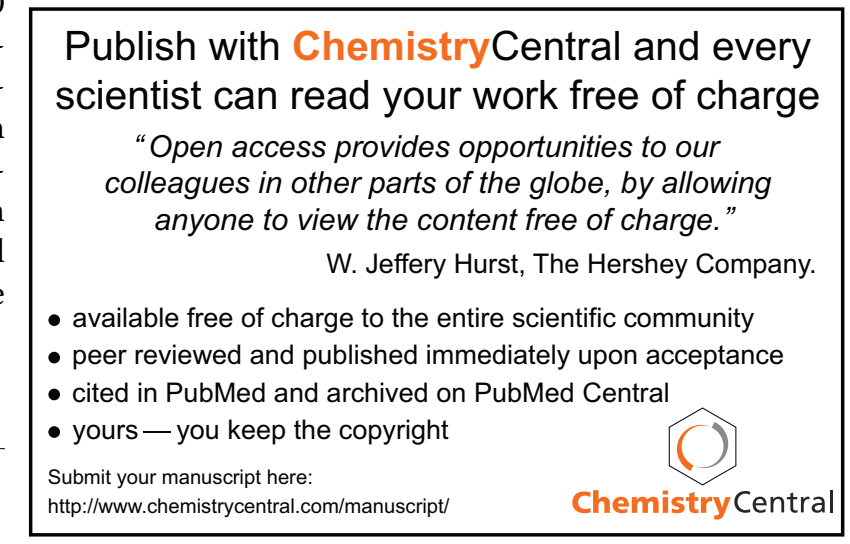

Published: 11 March 2014
References
1. Höck S, Riedl R: chem ${ }^{\mathrm{f}}$ : A purely functional chemistry toolkit. Journal of Cheminformatics 2012, 4:38.
2. Erwig M: Inductive Graphs and Functional Graph Algorithms. Journal of Functional Programming 2001, 11(5):467-492.

doi:10.1186/1758-2946-6-S1-P11

Cite this article as: Sosnin et al:: A new software for fragment-based

QSAR and its applications. Journal of Cheminformatics 2014 6(Suppl 1):P11.

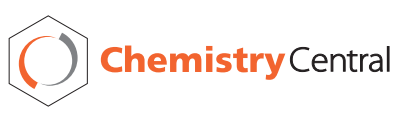

C 2014 Sosnin et al; licensee Chemistry Central Ltd. This is an Open Access article distributed under the terms of the Creative Commons Attribution License (http://creativecommons.org/licenses/by/2.0), which permits unrestricted use, distribution, and reproduction in any medium, provided the original work is properly cited. The Creative Commons Public Domain Dedication waiver (http://creativecommons.org/publicdomain/zero/1.0/) applies to the data made available in this article, unless otherwise stated. 